\section{Sommaire du plan stratégique 2004 de l'Association canadienne de gastroentérologie}

\author{
Ronald J Bridges MD FRCPC, président, ACG \\ William G Paterson MD FRCPC, président sortant, ACG \\ Paul Sinclair M. Sc., directeur général, ACG \\ David G Morgan MD FRCPC, président désigné, ACG
}

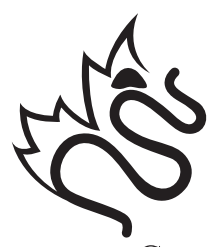
L'Association Canadienne
de Gastroentérologie

For English see page 164
$\mathrm{E}^{\mathrm{n}}$ 2004, l'Association canadienne de gastroentérologie (ACG) préparé un plan stratégique (1) pour s'appuyer sur les réalisations des 42 années de travail précédentes (2) et orienter ses progrès et son évolution au cours des cinq années suivantes. L'ACG a toujours fait la promotion de l'avancement de la science et de l'art de la gastroentérologie par le soutien de la formation et de l'éducation à tous les échelons et par l'appui et la participation à l'avancement de l'étude des sciences de la santé et des maladies digestives. Le plan stratégique était structuré en cinq grandes orientations, dotés d'objectifs et de cibles.

\section{LES GRANDES ORIENTATIONS DU PLAN STRATÉGIQUE 2004 DE L'ACG}

1. Privilégier l'essor de la prochaine génération de praticiens cliniques, de chercheurs, de professeurs et de chefs de file en gastroentérologie.

2. Élaborer et soutenir les affaires gouvernementales et les programmes de défense d'intérêts continus sur les scènes locale, provinciale et fédérale.

3. Promouvoir et améliorer les alliances professionnelles et les partenariats provinciaux dynamiques.

4. Soutenir et améliorer la portée de la pratique clinique et les programmes de formation et de recherche.

5. Mettre au point des ressources pour offrir des services supplémentaires et améliorés aux membres de l'ACG.

Au nom du conseil de l'ACG, nous sommes heureux de présenter un rapport résumant les activités et les réalisations de l'organisme à l'égard de ces grandes orientations depuis cinq ans.

\section{Grande orientation 1}

On a abattu beaucoup de travail par divers projets visant à privilégier l'essor de la prochaine génération de dispensateurs de soins, de chercheurs, de professeurs et de chefs de file en santé digestive. En réaction aux rapports sur les temps d'attente excessifs des patients pour recevoir des soins et aux inquiétudes quant au nombre de spécialistes en santé digestive, l'ACG a lancé un projet de planification des effectifs en 2004, pourvu de trois projets complémentaires. Le premier était un recensement détaillé des effectifs en gastroentérologie au Canada par rapport à celui d'autres pays et un rapport des professionnels dispensant des soins en santé digestive au Canada $(3,4)$. Le deuxième était un groupe consensuel multidisciplinaire responsable d'établir les temps d'attente maximaux ou les normes de temps d'attente selon les données probantes et les compétences en santé digestive (5). Le troisième était une série de vérifications de la pratique $(6,7)$, soit le programme de vérification de la pratique en gastroentérologie (PAGE) (8) et le sondage sur l'accès à la gastroentérologie (SAGE) (9), qui quantifiaient les véritables temps d'attente pour recevoir des consultations et des interventions en maladies digestives. En 2007, l'ACG a été invitée à faire partie de L'Alliance sur les temps d'attente (ATA). On a fait le compte rendu des effectifs d'endoscopie clinique nationaux et provinciaux dans une étude publiée en 2007 (4). Tous ces renseignements sont essentiels à la planification des effectifs pour des soins en santé digestive de qualité auprès d'une population croissante et vieillissante, compte tenu de l'évolution des normes des soins et des nouvelles technologies. En 2009, on a procédé à une évaluation des besoins de la recherche portant sur les perceptions des intervenants au sujet des investissements relatifs en recherche. L'information est en cours d'analyse. On a accompli des progrès considérables en matière de planification des effectifs. Cependant, il faudra réfléchir, planifier et agir davantage pour utiliser avec efficacité les données acquises afin d'aborder les organismes nationaux et provinciaux de formation, de financement et d'attribution de permis et de prôner un nombre pertinent de spécialistes en santé et de chercheurs dans le domaine de la santé et des maladies digestives.

Étant donné les membres actuels de l'ACG, les représentants de la profession semblent provenir largement de régions et milieux culturels variés. Les programmes des résidents de gastroentérologie en formation (GRIT) et des chercheurs-boursiers lors de la Semaine canadienne sur les maladies digestives (SCMD) ont réussi à mieux faire connaître la gastroentérologie aux stagiaires et, ces dernières années, des candidats de qualité ont été attirés par les programmes de résidence en gastroentérologie. L'ACG appuie un programme de partenariat fructueux avec les Instituts de recherche en santé du Canada (IRSC), la Fondation canadienne des maladies inflammatoires de l'intestin (FCMII), l'industrie et la Fondation canadienne pour la promotion de la santé digestive (FCPSD) afin de former des étudiants au premier cycle, aux études supérieures et au postdoctorat ainsi que de fournir des subventions d'exploitation aux membres. Un cours annuel destiné aux stagiaires, organisé en collaboration avec la FCMII, les IRSC et l'industrie, est précieux pour attirer des stagiaires dans le

L'ACG est fière de présenter ses bienfaiteurs:

Abbott Canada
AstraZeneca Canada Inc
Axcan Pharma Inc

Olympus Canada Inc 
domaine. Les nouvelles politiques et normes de gouvernance de l'ACG sont conçues pour assurer de la flexibilité dans ce domaine, afin de mieux permettre aux stagiaires, aux médecins et scientifiques intéressées de participer aux affaires de l'organisme.

Devant l'intérêt de la communauté et des associations professionnelles, on a créé, en partenariat avec la FCPSD, un nouveau prix de recherche destiné aux gastroentérologues communautaires, la bourse de recherche communautaire (GRC). Cette bourse est maintenant décernée tous les deux ans, en même temps que la bourse de formation des professionnels-cliniciens. Les bourses postdoctorales continuent de soutenir la formation des cliniciensscientifiques, mais ce secteur n'a pas pris d'expansion et le financement n'a pas augmenté pour favoriser des formations plus intensives et plus longues. Le financement de la recherche des résidents, des étudiants et des nouveaux chercheurs a été exemplaire ces dernières années, mais en raison de la situation économique actuelle, l'organisme devra faire preuve de créativité pour trouver d'autres sources de financement de la recherche. Les programmes de formation en gastroentérologie clinique ont été réduits dans certaines provinces, malgré le besoin établi de former plus de spécialistes en santé et maladies digestives. Il y a des possibilités de carrière en gastroentérologie, mais dans certaines régions du pays, des limites s'associent à la disponibilité des ressources d'endoscopie et du financement universitaire des postes de recherche universitaire. Les directeurs des programmes de formation en gastroentérologie ont assuré une promotion et un appui efficaces dans l'élaboration de meilleures normes pour les programmes de formation du Collège royal des médecins et chirurgiens du Canada (CRMCC).

\section{Grande orientation 2}

On a consacré beaucoup d'énergie et de ressources afin d'influencer et d'améliorer la prestation des soins en santé digestive. Dans le plan stratégique de 2004, il était indiqué que ce secteur devait susciter plus d'attentions et de mesures. L'ACG, membre active de l'ATA canadienne, a correspondu et interagi avec le gouvernement et le public au sujet des temps d'attente et de l'accès aux soins. De plus, l'organisme a réagi à plusieurs enjeux de réglementation, tels que l'exercice de pressions auprès de l'Agence canadienne des médicaments afin d'utiliser des médicaments existants et nouveaux pour le traitement des maladies digestives. Le comité de recherche et les membres de la communauté de la recherche de l'ACG ont participé au forum de l'Institut de la nutrition, du métabolisme et du diabète au sujet du plan stratégique des IRSC. Malgré ces activités, les progrès sont limités, et l'ACG n'a toujours pas attiré l'attention des gouvernements fédéral et provinciaux ni ne se fait entendre de l'ensemble du public canadien. Depuis cinq ans, l'ACG a joué un rôle accru pour préconiser les soins, l'assurance-qualité, la recherche et les ressources de formation en santé digestive. Pour maintenir la croissance et les succès dans ce secteur, il faut améliorer les relations, la pratique et l'information en recherche et travailler en collaboration avec la Fondation de l'ACG, les associations provinciales, les partenaires et les organismes affiliés. En collaboration avec la FCPSD, l'ACG a entrepris un processus en vue de planifier une stratégie nationale en santé digestive afin d'affronter la situation et, on l'espère, de défendre une position commune auprès du gouvernement et d'autres organismes. L'ACG, en partenariat avec la FCPSD, sa Fondation, est proactive pour tenter de susciter l'intérêt des décideurs à tous les échelons.

\section{Grande orientation 3}

Depuis cinq ans, l'ACG travaille à promouvoir et à améliorer les alliances professionnelles et les partenariats provinciaux. On a réalisé des progrès par la prestation de programmes de formation, d'accréditation de programmes, d'un programme de professeurs invités et d'initiatives coopératives d'évaluation des temps d'attente. Cependant, les soins sont surtout dispensés sur la scène provinciale, et l'organisme national a éprouvé de la difficulté à s'arrimer aux problèmes provinciaux. On a repéré d'autres moyens d'aider les organismes provinciaux, dans des domaines comme les sites Web et le soutien administratif, l'organisation de congrès régionaux et l'obtention de fonds de recherche pour les chercheurs communautaires afin de répondre à des problèmes précis liés à la pratique. La FCPSD représente également un lien important vers les organismes provinciaux, car elle fournit du précieux matériel d'éducation aux patients. Les activités doivent se poursuivre dans ce secteur.

L'ACG a également progressé dans ses efforts pour influer sur la gastroentérologie dans le monde. Elle a fourni de l'aide afin de livrer du matériel à des pays en voie de développement comme la Jamaïque, la Bolivie, l'Uruguay et Madagascar. Les fonds amassés grâce au World Congress of Gastroenterology de 2005, qui avait lieu à Montréal, au Québec, et qui a obtenu un franc succès, ont permis de créer la bourse de formation du fonds patrimonial, qui soutiendra des projets visant à améliorer les possibilités de formation pour des personnes de pays en voie de développement.

De plus, la bourse d'étude latino-américaine permet d'exposer des stagiaires de pays en voie de développement à des possibilités de recherche au Canada. L'ACG soutient également des stagiaires et des professeurs internationaux afin qu'ils participent au programme GRIT pendant la SCMD.

\section{Grande orientation 4}

Soutenir et améliorer la portée de la pratique clinique et les programmes de formation et de recherche constituent des priorités constantes de l'organisme depuis sa création en 1962. On a constaté une activité, des progrès et des succès considérables dans ces secteurs depuis cinq ans. L'ACG est un prestataire accrédité par le CRMCC pour le maintien du certificat (MDC) et est reconnue pour ses qualités de chef dans ce secteur. Le MDC est un service important pour les membres. Le portail de formation de l'ACG, qui a récemment été lancé en anglais (ePortal, www.cag-eportal. org), procure de nouvelles possibilités de formation, de vérification de la pratique, d'autoévaluation et de projets d'apprentissage personnel. On a mis en place un cyberprogramme d'assurance-qualité (10), découlant des programmes de vérification de la pratique PAGE et lié à la pratique de la coloscopie, qui permet aux membres de procéder à des évaluations au point de service et de comparer leur performance personnelle à celle de collègues, en temps réel. L'ACG a obtenu un appui solide du CRMCC pour ce programme, qui répond directement aux mandats du CRMCC d'offrir plus de mécanismes d'autoévaluation et d'excellentes méthodes pour repérer les besoins non perçus.

La série de conférences interactives a été remodelée pour assurer un meilleur accès à l'enregistrement de conférences en temps réel. Pour améliorer l'accès et la longévité d'excellent matériel de formation provenant de la SCMD et des congrès régionaux, l'ACG a investi dans une technologie pour enregistrer les conférences et les programmes et en permettre la diffusion sous de multiples formats dans son portail, y compris les vidéos et la baladodiffusion.

Des événements de formation pour les stagiaires très bien cotés, y compris les séances scientifiques par vidéo sur l'heure du midi et les programmes GRIT et aux chercheurs-boursiers pendant la SCMD, ont continué de prendre de l'expansion. Ils permettent aux stagiaires de s'exposer à la spécialité et d'assister à des cours interactifs de qualité avec des médecins, des scientifiques et des collègues de partout au pays. 
La SCMD continue de progresser et est perçue comme une tribune de formation de qualité orientée par un sondage annuel sur les besoins de formation des membres par des évaluations officielles des besoins. Elle est reconnue comme un congrès de choix pour les gastroentérologues en exercice au Canada.

Le Journal canadien de gastroentérologie a crû et évolué pour devenir une revue clinique qui soutient grandement la recherche clinique au Canada. L'augmentation régulière de son facteur d'impact depuis cinq ans témoigne du dévouement et de l'engagement du comité de rédaction.

L'ACG accorde un soutien limité aux congrès régionaux sur la scène locale. Cependant, l'ACG n'organise pas de congrès cliniques ou de recherche régionaux, ce que les membres perçoivent comme une carence. Des mesures sont prises pour travailler avec les organismes provinciaux afin d'offrir des congrès régionaux qui soutiendront mieux la communauté de la gastroentérologie.

L'ACG a subventionné la création et la diffusion de plusieurs guides de pratique clinique $(5,11-14)$ depuis quelques années, qui ont été bien reçus et ont influé sur les soins en santé digestive.

L'évolution de la FCPSD comme véhicule de collecte de fonds pour la recherche a un potentiel énorme pour les membres de l'ACG.

On a créé des bourses pour saluer et promouvoir l'excellence dans les secteurs clinique, de l'enseignement et de la recherche. Des bourses pour les jeunes professeurs, l'excellence en enseignement, les jeunes chercheurs, l'excellence en recherche et les professeurs invités soulignent la réussite et contribuent à rehausser les soins et la science en santé digestive au pays.

Il demeure nécessaire d'élaborer et d'utiliser les technologies informatiques pour contribuer aux projets de recherche, y compris les essais cliniques et les bases de données de projets d'assurancequalité.

\section{Grande orientation 5}

En 2009, l'ACG a terminé une analyse complète de sa structure organisationnelle, qui a donné lieu à la mise à jour de règlements généraux désormais soutenus par des politiques de gouvernance. Le nouveau modèle se veut la base pour élaborer des ressources afin d'offrir des services supplémentaires et améliorés aux membres de l'ACG. L'ACG s'inspire du code d'éthique de l'Association médicale canadienne, auquel elle adhère, et s'est munie de principes et de lignes directrices pour ses interactions avec l'industrie. Depuis cinq ans, les commandites et les partenariats avec l'industrie ont pris de l'expansion, mais il est peu probable que cette tendance se poursuivre. Les états financiers annuels vérifiés confirment que l'organisme est dans une situation financière solide et que ses réserves sont suffisantes pour exploiter le bureau de l'ACG pendant un an et soutenir un congrès annuel (SCMD).

La revitalisation et la restructuration de la FCPSD constituent une étape importante vers l'atteinte de fonds de dotation stables pour soutenir les bourses de recherche et de formation. En plus de mieux faire connaître la santé et les maladies digestives, la Fondation a le potentiel important de recueillir des fonds pour appuyer des programmes nationaux de recherche et d'éducation publique. Les activités récentes ont porté sur la mise sur pied d'une stratégie nationale en santé digestive afin de mieux faire connaître la santé et les maladies digestives et d'obtenir de nouvelles sources de financement. On a pris des mesures importantes, mais il reste beaucoup de travail à accomplir.

\section{SOMMAIRE}

L'ACG a réalisé des gains importants depuis cinq ans. Elle demeure vouée au perfectionnement professionnel, à la recherche et aux soins des patients dans tous les secteurs de la santé et des maladies digestives, en appui à la santé économique et sociale de tous les Canadiens. Tous les objectifs et les cibles liés aux grandes orientations du plan stratégique de 2004 ont été abordés, et la plupart ont été réalisés en totalité ou en partie. L'organisme est actif dans plusieurs secteurs puisqu'il maintient le dialogue avec des partenaires, renforce des programmes en place, repère les secteurs où les objectifs et les cibles ne sont pas atteints et crée de nouvelles initiatives. Ces activités visent à offrir de la valeur à vous, les membres, grâce à l'amélioration des programmes en santé et maladies digestives. Nous pensons que l'ACG est bien placée pour répondre aux occasions et aux défis des prochaines années. À cet effet, un nouveau plan stratégique quinquennal est en cours d'élaboration et sera présenté aux membres dans un avenir rapproché. La direction de l'ACG accueillera vos commentaires et vos suggestions pour améliorer l'évolution et l'exploitation continues de l'organisme.

\section{RÉFÉRENCES}

1. Sherman PM, Fedorak RN, Leddin D, Wallace JL. Canadian Association of Gastroenterology 2004 Strategic Plan. Can J Gastroenterol 2004;18:125-6.

2. Fedorak R, Sherman P. The 1993 Canadian Association of Gastroenterology Strategic Plan: Excellence in achievement. Can J Gastroenterol 2003;17:683-4.

3. Moayyedi P, Tepper J, Hilsden R, Rabeneck L. International Comparisons of Manpower in Gastroenterology. Am J Gastroenterol 2007;102:478-81.

4. Hilsden RJ, Tepper J, Moayyedi P, Rabeneck L. Who provides gastrointestinal endoscopy in Canada? Can J Gastroenterol 2007;21:843-6.

5. Paterson WG, Depew WT, Pare P et coll; for the Canadian Association of Gastroenterology Wait Time Consensus Group. Canadian consensus on medically acceptable wait times for digestive health care. Can J Gastroenterol 2006;20:411-23.

6. Armstrong D, Hollingworth R, Gardiner T et coll. Practice Audit in Gastroenterology (PAGE) Program: A novel approach to continuing professional development. Can J Gastroenterol 2006;20:405-10.

7. Leddin D, Armstrong D, Barkun AN et coll. Access to specialist gastrenterology care in Canada: Comparison of wait times and consensus targets. Can J Gastroenterol 2008;22:161-7.

8. Armstrong D, Barkun AN, Chen Y et coll. Access to specialist gastroenterology care in Canada: The Practice Audit in Gastroenterology (PAGE) wait times program. Can J Gastroenterol 2008;22:155-60.

9. Leddin D, Bridges RJ, Morgan DG et coll. Survey of Access to GastroEnterology in Canada: The SAGE wait times program. Can J Gastroenterol 2010;24:20-5.

10. Hollingworth R, Dube C. The Canadian Association of Gastroenterology Endoscopy Quality Initiative: Leading the wave. Can J Gastroenterol 2009;23:506-9.

11. Armstrong D, Marshall JK, Chiba N et coll. Canadian Consensus Conference on the management of gastroesophageal reflux disease in adults - update 2004. Can J Gastroenterol 2005;19:15-35.

12. Rostom A, Moayyedi P, Hunt R. Canadian consensus guidelines on long-term nonsteroidal anti-inflammatory drug therapy and the need for gastroprotection: Benefits versus risks. Aliment Pharmacol Ther 2009;29:481-96.

13. Sadowski DC, Bernstein CN, Bitton A et coll. Canadian Association of Gastroenterology Clinical Practice Guidelines: The use of tumour necrosis factor-alpha antagonist therapy in Crohn's Disease. Can J Gastroenterol 2009;23:185-202.

14. Barkun AN, Bardou M, Kuipers EJ et coll. International consensus recommendations on the management of patients with nonvariceal upper gastrointestinal bleeding. Ann Int Med 2010;152:101-13. 


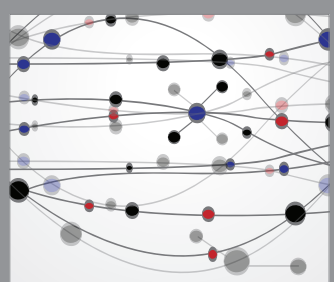

The Scientific World Journal
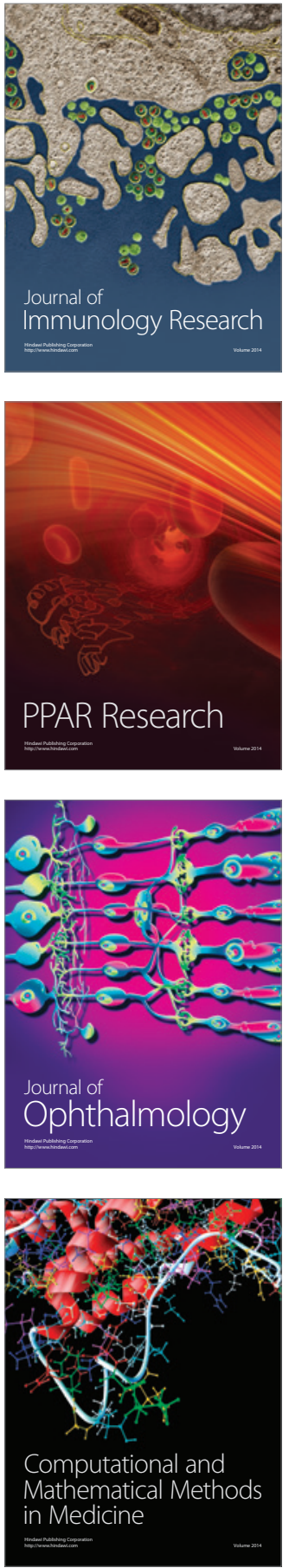

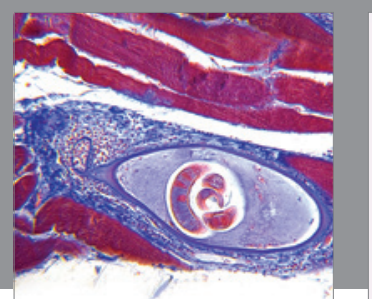

Gastroenterology Research and Practice

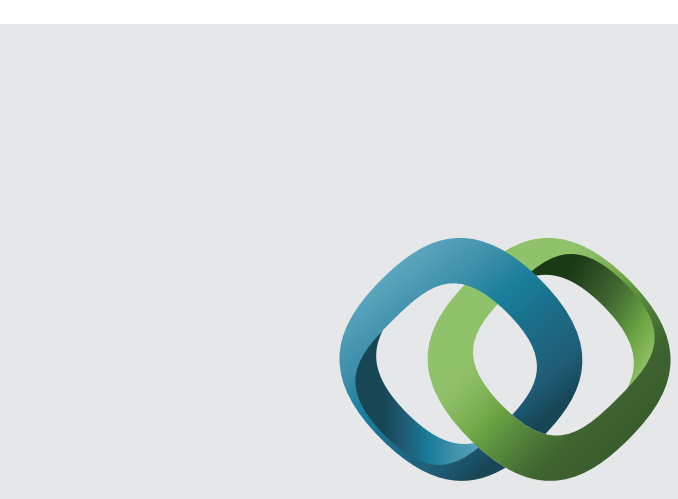

\section{Hindawi}

Submit your manuscripts at

http://www.hindawi.com
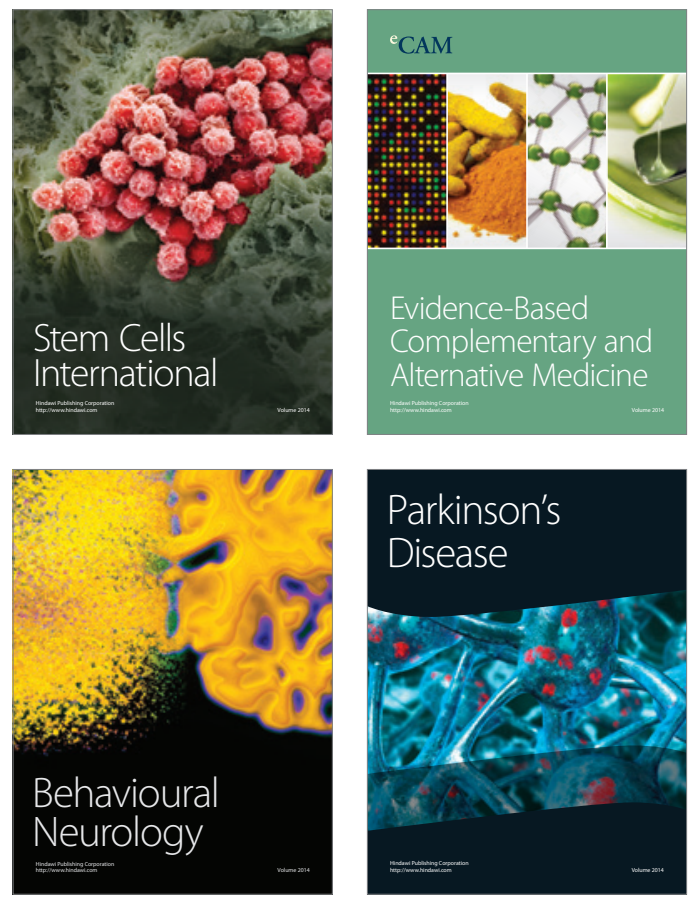
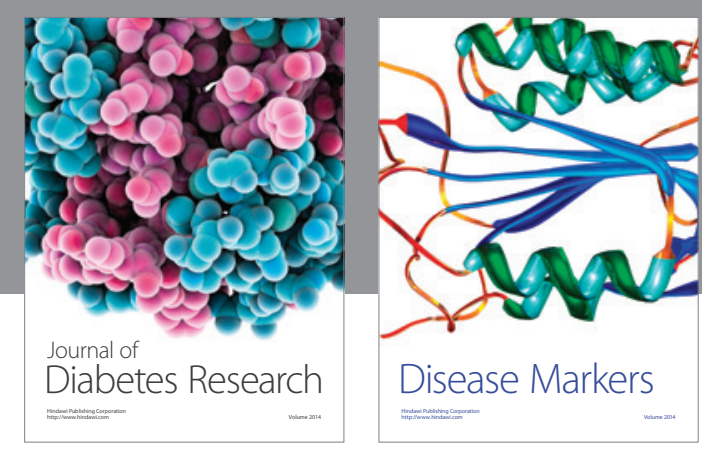

Disease Markers
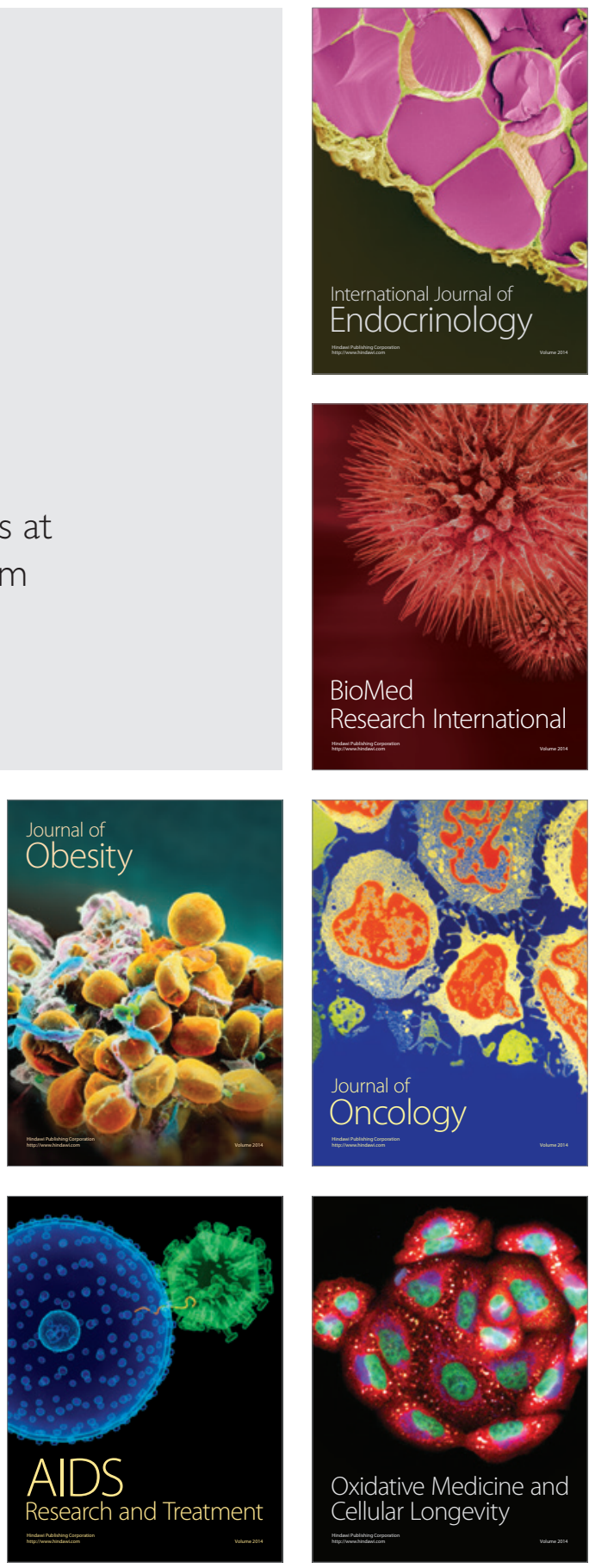